\title{
A New Detection Metric for EDS Detectors
}

\author{
Patrick P. Camus ${ }^{1}$
}

1. EDAX Inc, 91 McKee Place, Mahwah, NJ, USA.

When comparing the detection capabilities of EDS detectors, the primary detection metric of interest that is implied but not always stated is the total x-ray detection, approximated by:

$\mathrm{TXD} \sim$ emitted $\mathrm{x}$-rays from sample * SEM environment transmission * detector efficiencies * where geometric efficiency

detector efficiencies $\sim$ window materials transmission * window support transmission * detector environment transmission * sensor efficiency

With the same sample and SEM conditions, the detector efficiencies are assumed to be the same, so the geometric efficiency is the presumed prime metric. The core parameter in this metric is the solid angle of the detector which can be calculated very easily [1], but can be approximated as:

$\mathrm{SA}=$ area $/(\text { detector-to-sample-distance })^{\wedge} 2$

Historically, this metric provides the user with a good indication of the x-ray input performance that is expected for a given microscope installation, and can be provided by the manufacturer. In addition, the area of the detector sensor is a simple number for the user to interpret.

These simple metrics were very useful for SiLi detectors and early generation silicon-drift detectors using polymer windows from a single manufacturer. But generational changes in detector and window technologies make SA and detector area values important contributions and do not consider enough design variations. A new metric which includes all of the potential variations should be considered.

An example was found while comparing the performance of the older generation EDAX Octane 60 SDD with a polymer window and the newest EDAX Elite 30 SDD with a silicon-nitride window, both at the same SEM geometry. It was found that the input and output x-ray count rates were significantly higher for the smaller SDD when normalized for area, Figure 1. This observation was hard to believe using the previous assumptions of similarity of the detector module designs. But these SDDs have significantly different sensor fabrication techniques and widely different window designs. This paper investigates how the design of many generations of detectors affect the detector efficiencies, indicating the need for a new detection metric.

A number of detector designs were targeted for modelling, Table 1. The transmission of each material layer as a function of the incoming x-ray energy was calculated using the Filter Transmission web site of The Center for X-Ray Optics, Lawrence Berkeley National Laboratory's (LBNL) [2]. The greatest energy variation in the transmission curves was found in the window efficiency plots, more specifically in the window support grid plot, Figure 2. The superior transmission of the grid used for the siliconnitride window permits up to $20 \%$ increase in all $\mathrm{x}$-rays from $\sim 5 \mathrm{keV}$ up to $\sim 20 \mathrm{keV}$. This leads to a significantly higher TXD for those sensors than would be expected for other sensors. In this case, the SA is not the best metric for predicting a detector with a higher TXD value. 
A new metric is proposed that contains both the geometric efficiency but also the TXD. The proposal is to define the total $\mathrm{x}$-ray detection efficiency (TXDE) as:

$\mathrm{TXDE}=\mathrm{TXD} / \mathrm{sec} / \mathrm{nA} / \mathrm{sR}$

Where the TXD is for a defined material and SEM operating condition.

With this proposal, there is not simply a single metric, but there could be a select few important metrics used for comparison. An obvious metric could be the analysis of pure copper (from a grid or tape used for insulating materials) or a standardized stainless steel collected at $20 \mathrm{keV}$.

Comparing values from different detectors using this metric would provide a much more useful information for a user than the simplistic detector area.

References:

[1] http://tpm.amc.anl.gov/NJZTools/XEDSSolidAngle.html.

[2] http://henke.lbl.gov/optical_constants/filter2.html.

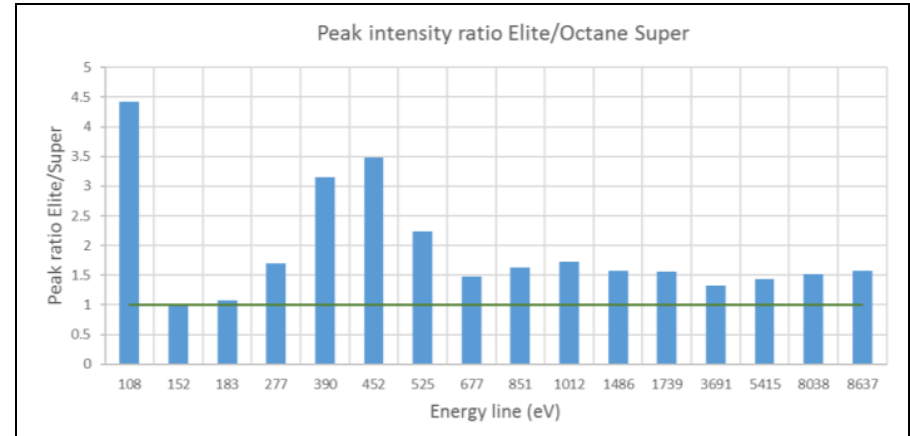

Figure 1. Plot of area normalized $x$-ray intensity ratios for 2 generations of EDAX SDD.

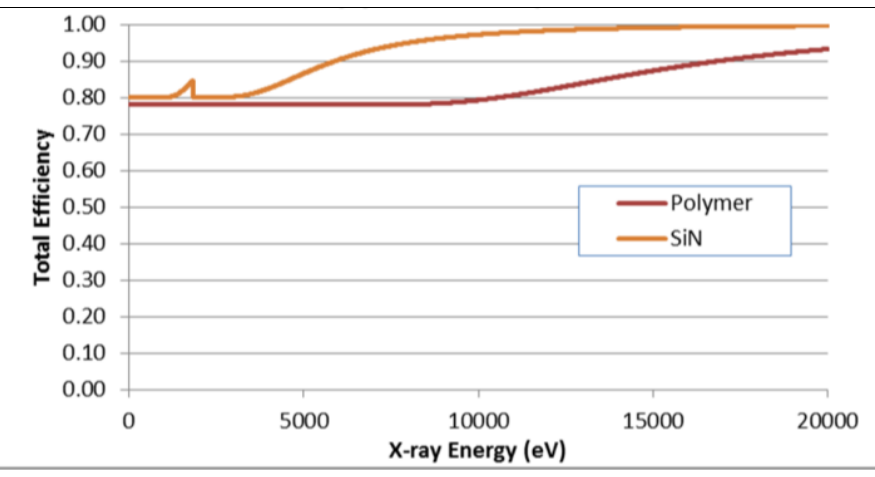

Figure 2. X-ray transmission efficiency plots for 2 types of EDS thin window grid support structures.

Table 1. Description of materials and thicknesses of the modelled detector modules.

\begin{tabular}{|c|c|c|c|c|c|c|c|c|c|}
\hline & Sensor & & & & & Window (um & & Support & \\
\hline $\mathrm{SiLi}+\mathrm{Be}$ & $10 \mathrm{~mm} 2$ & $3 \mathrm{~mm} \mathrm{Si}$ & $\mathrm{DL}=0.08 \mathrm{Si}$ & $0.01 \mathrm{Ni}$ & vacuum & $10 \mathrm{Be}$ & & $100 \%$ & \\
\hline SiLi + polymer & $10 \mathrm{~mm} 2$ & $3 \mathrm{~mm} \mathrm{Si}$ & $\mathrm{DL}=0.08 \mathrm{Si}$ & $0.01 \mathrm{Ni}$ & vacuum & 0.3 polymer & $0.04 \mathrm{Al}$ & $76 \%$ & 380um S \\
\hline $\begin{array}{l}\text { SDD1 + } \\
\text { polymer }\end{array}$ & $28 \mathrm{~mm} 2$ & $\begin{array}{c}0.45 \mathrm{~mm} \\
\mathrm{Si}\end{array}$ & $\begin{array}{c}\mathrm{DL}=0.03 \\
\mathrm{SiO} 2\end{array}$ & $0.04 \mathrm{Al}$ & $1 \mathrm{~mm} 1 \mathrm{~Pa} \mathrm{~N} 2$ & 0.3 polymer & $0.04 \mathrm{Al}$ & $76 \%$ & 380um S \\
\hline SDD2 + SiN40 & $17 \mathrm{~mm} 2$ & $0.5 \mathrm{~mm} \mathrm{Si}$ & $\begin{array}{c}\mathrm{DL}=0.03 \\
\mathrm{SiO} 2\end{array}$ & $0.04 \mathrm{Al}$ & & 0.04 Si3N4 & $0.04 \mathrm{Al}$ & $80 \%$ & 20um Si \\
\hline SDD2 + SiN20 & $17 \mathrm{~mm} 2$ & $0.5 \mathrm{~mm} \mathrm{Si}$ & $\begin{array}{c}\mathrm{DL}=0.03 \\
\mathrm{SiO} 2\end{array}$ & $0.04 \mathrm{Al}$ & vacuum & $0.02 \mathrm{Si} 3 \mathrm{~N} 4$ & $0.04 \mathrm{Al}$ & $80 \%$ & 20um Si \\
\hline $\begin{array}{l}\text { SDD1 } \\
\text { windowless }\end{array}$ & $28 \mathrm{~mm} 2$ & $\begin{array}{c}0.45 \mathrm{~mm} \\
\mathrm{Si}\end{array}$ & $\begin{array}{c}\mathrm{DL}=0.03 \\
\mathrm{SiO} 2\end{array}$ & $0.04 \mathrm{Al}$ & vacuum & 0 & 0 & $100 \%$ & 0 \\
\hline
\end{tabular}

